\title{
NOTE ON SUBSTITUTION GROUPS OF EIGHT LETTERS.
}

BY G. A. MILLER, PH.D.

I HAVE found a non-primitive group of degree eight and order twenty-four which is not contained in Professor Cayley's list, vol. 25, p. 140, Quarterly Journal of Mathematics, nor in Professor Cole's supplementary list published in the Bulletis, vol. 2, pp. 187 and 189. The group may be written in the form

$$
(a b c d \cdot e f g h) \text { pos. }(a e . b g \cdot c f \cdot d h) \text {. }
$$

The substitutions are

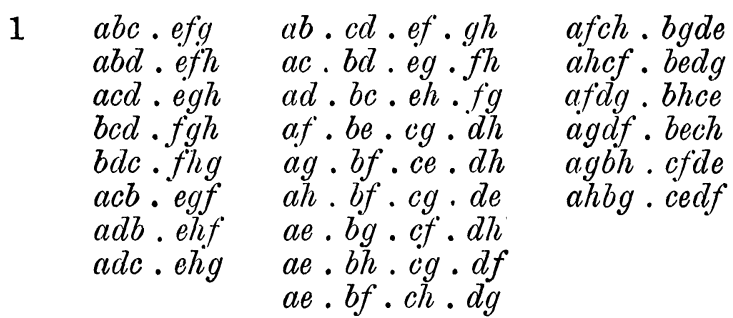

Two of the six groups of order 16 given by Professor Cole in the Bulletin, vol. 2 , p. $18 \%$, are identical, viz. :

\begin{tabular}{ll|ll}
1 & $a c \cdot b d$ & 1 & $e g \cdot f h$ \\
$a c$ & $b d$ & $e f \cdot g h$ & $e h \cdot f g$ \\
$a b . c d$ & $a d \cdot b c$ & $e f g h$ & $e h g f$ \\
$a b c d$ & $a d c b$ & $e g$ & $f h$
\end{tabular}

and

\begin{tabular}{ll|lll}
1 & $a c \cdot b d$ & 1 & $e g \cdot f h$ \\
$a c$ & $b d$ & $e f g h$ & $e h g f f$ \\
$a b \cdot c d$ & $a d \cdot b c$ & $e g$ & $f h$ \\
$a b c d$ & $a d c b$ & & $g h$ & $e h \cdot f g$
\end{tabular}


The substitutions of the group of order 12, given just below these six, should be

1

$$
\begin{array}{ll}
a b c \cdot e f g & a b \cdot c d \cdot e f \cdot g h \\
a c b \cdot e g f & a c \cdot b d \cdot e g \cdot f h \\
a b d \cdot e f h & a d \cdot b c \cdot e h \cdot f g \\
a d b \cdot e h f & \\
a c d \cdot e g h & \\
a d c \cdot e h g & \\
b c d \cdot f g h & \\
b d c \cdot \text { fhg } &
\end{array}
$$

Since two of the published groups of degree eight are identical and a new one is added, their total number is still 199.

It remains to note a typographical error in Professor Cole's supplementary list. The combining substitution in the third group of order 32, p. 188, should be aecg. bf. dh instead of aecg. $b f d h$; the latter substitution gives the same group as ae. $b f . c g . d h$, which is already in the list. There is a considerable number of other typographical errors in each of the lists referred to, but they are of such a nature as to be easily detected by those employing the groups and require therefore no special mention.

Ann ArBor, March 12, 1894.

With the above corrections, for which I am under great obligations to Dr. Miller, the question of the possible substitution groups of eight letters is now settled beyond doubt.

F. N. CoLE. 